\title{
Generation of Nanometer Wavelength Acoustic Waves
}

\author{
O.Yu. Komina ${ }^{1}$, M.E. Adamova ${ }^{1}$, E.A. Zhukov ${ }^{1}$, A.P. Kuz'menko², V.I. Zhukova ${ }^{3}$ \\ 1 Pacific National University, 136, Tihookeanskaja st., 680035 Khabarovsk, Russia \\ 2 Southwest State University, 94, 50 Let Octyabrya st., 305040 Kursk, Russia \\ ${ }^{3}$ Far Eastern State Transport University, 47, Serysheva st., 680021 Khabarovsk, Russia
}

(Received 18 June 2016; published online 29 November 2016)

\begin{abstract}
The possibility of acoustic wave generation of nanometer range in plates is shown. The experimental results that show the possible reconfiguring of the generator frequency in $\mathrm{YFeO}_{3}$ with a constant magnetic field are given.
\end{abstract}

Keywords: Yttrium orthoferrite, Acoustic waves, Domain wall, Lamb waves.

\section{INTRODUCTION}

Earlier it was shown that the motion of the domain wall (DW) in yttrium orthoferrite $\left(\mathrm{YFeO}_{3}\right)$ is accompanied by the generation of acoustic waves [1]. On the basis of this effect, the possible creation of the hypersonic wave generator was shown $[2,3]$. For the generator frequency control, a constant magnetic field which influences the DW speed can be used [4].

The weak ferromagnet $\mathrm{YFeO}_{3}$ that features transparency and high magneto-optical $\mathrm{Q}$ factor in the visible is one of the model crystals for studying DW dynamics [5]. The influence of a variable magnetic field on the $\mathrm{DW}$ dynamics in $\mathrm{YFeO}_{3}$ is well studied, but the influence of a constant field is not considered. There are separate experimental works on research of influence of the constant field in $\mathrm{YFeO}_{3}$ [6] and $\mathrm{FeBO}_{3}$ [4] crystals and also in ferrite garnet films [7]. We do not have information on theoretical works studying such influence in $\mathrm{YFeO}_{3}$. The available models [8] are inapplicable to $\mathrm{YFeO}_{3}$.

\section{GENERATION OF HYPERSONIC WAVES}

According to [9], magneto- acoustic interaction makes the speeds of a quasi-acoustic family of normal waves lower mainly in the low-frequency range. This leads to the emergence of certain speeds for shear elastic waves in $\mathrm{YFeO}_{3}$ at which magneto-acoustic interaction is compensated by the negative dispersion of speed for one of the normal modes [3].

Dependence of possible frequencies $f$ of a source on plate thickness is given in Fig. 1, the modes are enumerated from above. As the plate thickness is reduced, modes of hypersonic waves move to the area of high spatial frequencies. Dependences of the generated wave parameters on plate thickness can be used for the creation of the reconfigurable source of hypersonic vibrations. For ferromagnetic materials, the moving domain wall can be such external source, and when there is no dispersion, acoustic modes at the frequencies depending on DW speed will be generated [1]. As is seen from Fig. 1, the frequency of the generated waves can reach terahertz range, which corresponds to the nanometer range of wavelengths.

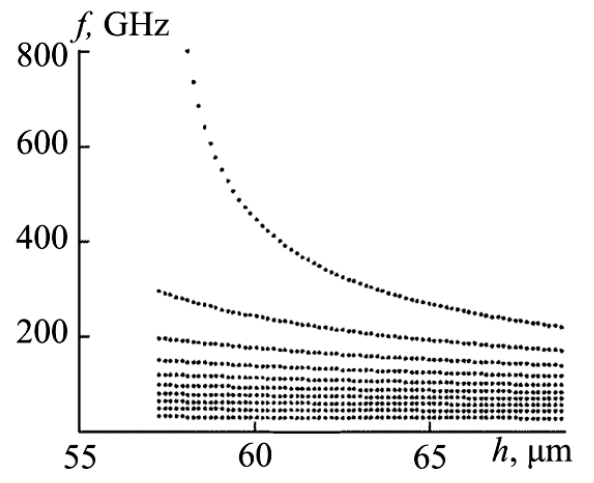

Fig. 1 -Dependence of frequency of generated hypersonic waves on plate thickness

Lack of dispersion because of the existence of longitudinal and transverse waves is also possible in nonmagnetic plates for Lamb waves. The corresponding dispersive equations for an isotropic plate are the following [10]:

$$
\operatorname{th}\left(\frac{\kappa_{t} h}{2}\right) / \operatorname{th}\left(\frac{\kappa_{l} h}{2}\right)=\left(\left(k^{2}-\kappa_{t}^{2}\right)^{2} / 4 k^{2} \kappa_{l} \kappa_{t}\right)^{+1}
$$

where $\kappa_{l}=\sqrt{k^{2}-\omega^{2} / s_{l}{ }^{2}}, \kappa_{t}=\sqrt{k^{2}-\omega^{2} / s_{t}{ }^{2}}, \omega$ is angular frequency, $k$ is the projection of the wave vector on $x$ axis, $h$ is plate thickness, $s_{l}$ and $s_{t}$ are longitudinal and transversal speeds of sound. The positive exponent in (1) corresponds to antisymmetric $\left(A_{i}\right)$ waves, the negative one -to symmetric $\left(S_{i}\right), i$ is the mode number. The calculation was conducted within the range $k=0-10^{4}$ $\mathrm{cm}^{-1}$ for the following parameters: $h=0.01 \mathrm{~cm}, s_{t}=4.2$ $\mathrm{km} / \mathrm{s}, s_{l}=7 \mathrm{~km} / \mathrm{s}$.

Fig. 2 shows for the modes $A_{2}, S_{2}, S_{3}$ and $A_{6}$, the frequency spectrum of phase $(v=\omega / k)$ and group $\left(v_{g r}=d \omega / d k\right)$ velocities. Solid lines correspond to $v_{g r}(\omega)$, dashed lines - to $v(\omega)$.

As it is seen from Fig. 2, at certain frequencies $v_{g r}$ and $v$ are maximally close to each other, which can be also used for generation of Lamb waves by an external source the role of which in nonmagnetic medias may be played by laser radiation. For $\mathrm{FeBO}_{3}$ in work [11] it is shown that the frequency of the generated waves also reaches terahertz range.

Nonlinearity of dispersive dependences with respect 
to plate parameters follows from the equations (1) [10].

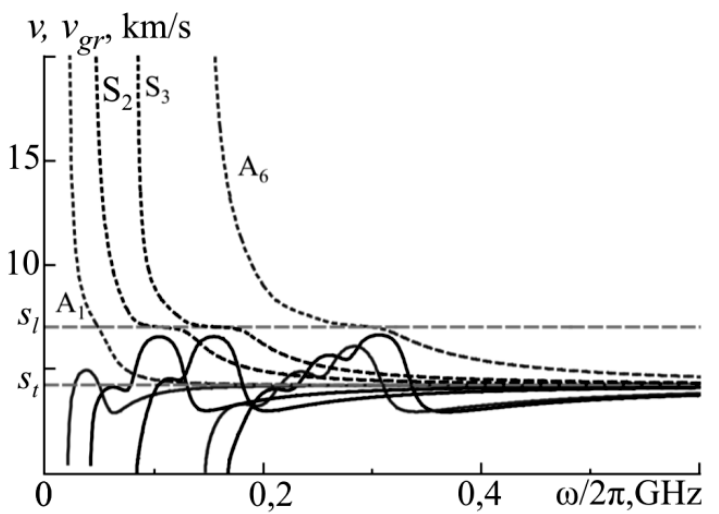

Fig. 2-Spectrum of phase and group velocities of Lamb waves

Fig. 3 shows the dependences $v_{g r}(k)$ and $v(k)$ as theplate thickness $h$ is reduced by $20 \%$. In Fig. 3, a it is seen that dispersive curves move to the short-wave range when the plate thickness is reduced. Expansion of maxima of group speed and growth of the frequencies corresponding to them is characteristic of dependences $v_{g r}(k)$ (Fig. 3, b). Also, the thickness variation affects the modes of various orders differently. Least of all it affects the behavior of the lowest modes.
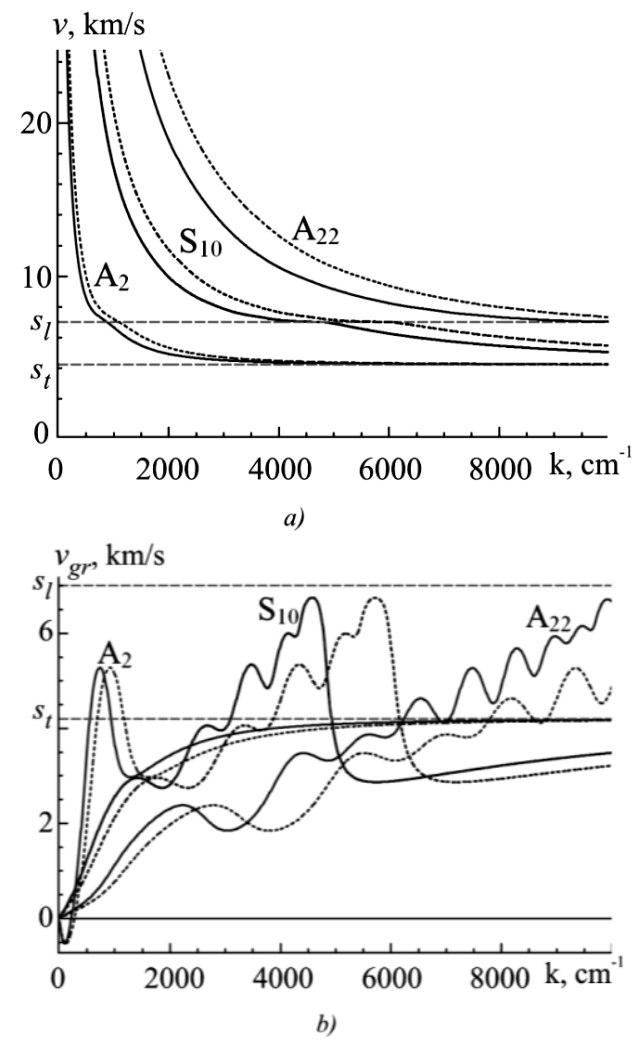

Fig. 3 - Effect of the plate thickness on $v(k)(a)$ and $v_{g r}(k)(b)$. Solid lines $-h_{1}=0.01 \mathrm{~cm}$, dashed lines $-h_{2}=0.008 \mathrm{~cm}$

Dependence of normal waves speeds on plate thickness can be used for the generator frequency reconfiguring. For this purpose, the constant magnetic field can also be used.

\section{INFLUENCE OF A CONSTANT MAGNETIC FIELD ON DW VELOCITY}

The scheme of experimental setup is given in [12]. The effect of a constant magnetic field on the DW speed was investigated by a technique $[1,6]$ in a $\mathrm{YFeO}_{3}$ plate of $2 \times 2 \mathrm{~mm}^{2}$ in size and about $100 \mu \mathrm{m}$ thick, cut perpendicular to an optical axis. To produce the external field, the permanent magnet was used.

The dependence of the DW velocity amplitude $V_{m}$ as a function of the driving sinusoidal magnetic field amplitude $H_{m}$ with a frequency $f=1 \mathrm{MHz}$ was obtained with a constant magnetic field oriented parallel to the plate along the DW motion and at the angle of $\approx 50^{\circ}$ to the sample plane close to the optical axis [3] (Fig. 4). Fig. 5 shows the dependencies $V_{m}$ on frequency $f$ of the driving field with an amplitude $H_{m}=48 \mathrm{Oe}$.

Here notions are introduced: - without a constant field, the constant magnetic field $H=18 \mathrm{Oe}$ is applied at an angle and $\boldsymbol{\Lambda}$ - parallel to the sample plane.

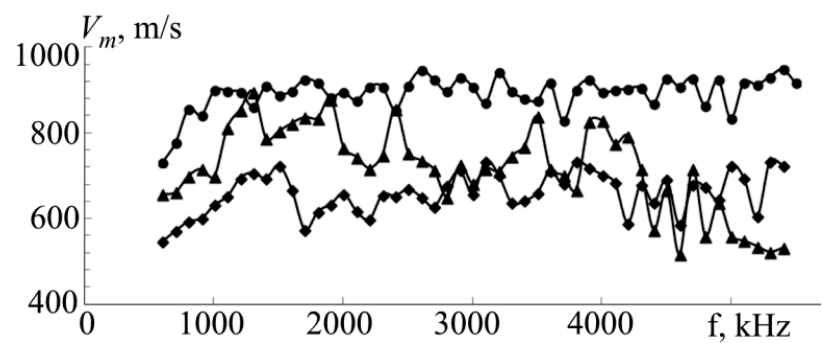

Fig. 4 - Dependence $V_{m}\left(H_{m}\right)$

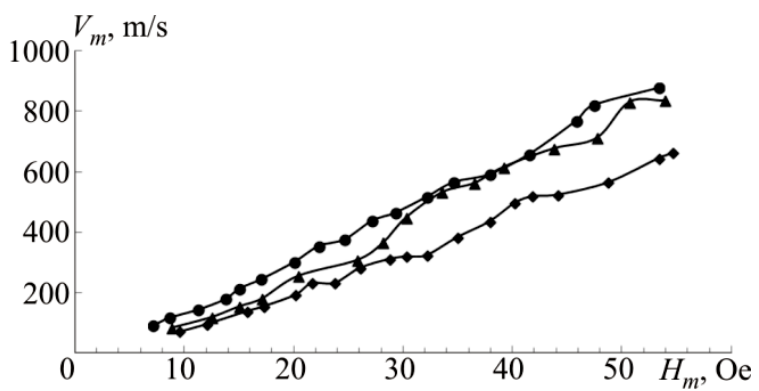

Fig. 5 - Dependence $V_{m}(f)$

It is seen from Fig. 4 that the velocity amplitude increases linearly with increase of the driving field amplitude according to [5]; the nature of dependence $V_{m}$ $\left(H_{m}\right)$ does not change when the constant field is applied.

The velocity amplitude increases monotonously with an increase in frequency $f$ of the driving field [5], and when $f$ reaches some value $f \approx 160 \mathrm{kHz} V_{m}$ remains approximately constant. Dependence in Fig. 5 corresponds to a range of $600-5500 \mathrm{kHz}$ where there is no such increase. It is seen that $V_{m}$ is not constant in both cases: when there is no constant magnetic field and also when it is applied along the DW motion. However, when the field is applied at some angle, the values of $V_{m}$ become almost equal; speed is stabilized. Besides, in all cases of the field application the speed increases. In doing so, the maximum increase is observed when the constant field is applied at an angle. 


\section{CONCLUSION}

The present work shows the possibility of the creation of nanometer range acoustic wave generator at the motion of DW with the set speed.

The specialties in calculated Lamb waves spectra show the possibility of wave generation by an external source. Influence of a sample thickness on the characteristics of hypersonic wave propagation in a plate is investigated.

\section{REFERENCES}

1. V.G. Bar'yakhtar, B.A. Ivanov, M.V. Chetkin. Phys.-Usp. 146 No 3, (1985).

2. E.A. Zhukov, V.I. Zhukova, A.V. Kaminsky, V.V. Korchevsky, V.I. Rimlyand. Bulletin of the Pacific State University. 26 No 3, (2012).

3. A.P. Kuzmenko, E.A. Zhukov, V.I. Zhukova, Tsz. Li, A.V. Kaminskii. Phys. Met. Metallogr. 106 No 2, 106 (2008).

4. M.E. Adamova, E.A. Zhukov, A.V. Kaminsky, O.Yu. Komina, A.P. Kuz'menko, Yu.I. Shcherbakov, J. Nano-Electron. Phys. 6 No 3, 03053 (2014).

5. P.V. Abakumov, A. P. Kuzmenko, A. N. Chaplygin, News of Kursk State University, 33 No 4, (2010).
Experimental results show that the constant magnetic field increases the value of DW velocity amplitude in yttrium orthoferrite and makes DW stabilized. The effect of a constant magnetic field on the DW velocity can be used for frequency reconfiguring of the generator.

The work was done at the financial support of the Pacific National University.

6. O.Yu. Komina, E.A. Zhukov, Yu.I. Scherbakov. Bulletin of the Pacific State University 40 No 1, (2016).

7. M.V. Chetkin, Yu.N. Kurbatova, T.B. Shapayeva. Solid State Phys. 52 No 9, (2010).

8. V.V. Volkov, V.F. Bokov. Solid State Phys. 50 No 2, (2008).

9. Y. Gulyaev, et al., Phys.-Usp. 40 No 7, (1997).

10. K. Graff, Dover Publications (1991).

11. E.A. Zhukov, M.E. Adamova, A.V. Kaminsky, A.P. Kuz'menko, V.I. Zhukova. J. Nano- Electron. Phys. 7 No 404092 (2015)

12. O.Yu. Komina, E.A. Zhukov, Yu.I. Scherbakov, A.V. Kaminsky, Scientific Notes of TOGU $\mathbf{5}$ No 1, 222 (2014). 